\title{
Novos desafios em SI: A crescente importância dos processos ciber-físicos
}

\author{
Silvia Fernandes ${ }^{1}$ \\ sfernan@ualg.pt \\ ${ }^{1}$ Faculdade de Economia \& CIEO, Research Centre for Spatial and Organizational Dynamics, Universidade \\ do Algarve, Campus de Gambelas, 8005-139 Faro, Portugal
}

DOI: 10.17013/risti.30.51-61

\begin{abstract}
Resumo: Os modelos de negócio e serviços têm sido desafiados pelos novos sistemas ciber-físicos, que combinam ambiente físico com digital. Estes têm levado a repensar os sistemas de informação (SI) e a arquitetura de processos. As empresas devem organizar o seu portefólio de SI de modo a conceber processos inteligentes que respondam rápida e criativamente aos novos desafios de trabalho e mercado. Uma arquitetura aberta, ágil, ativa e permeável ao contexto será muito útil. Se permitir serviços e interfaces bem concebidas, será possível uma correspondência contínua com as expetativas dos utilizadores e atividades resultando maior integração e personalização.
\end{abstract}

Palavras-chave: sistemas ciber-físicos, portefólio de SI, arquitetura de processos, arquitetura empresarial, indústria 4.

\section{New challenges in IS: The increasing importance of cyber-physical processes}

\begin{abstract}
Business models and services have been challenged by new cyberphysical systems that combine physical and digital environments. These have led to rethink information systems (IS) and process architecture. Companies should organize their IS portfolio to design intelligent processes that respond quickly and creatively to new job and market challenges. An open, agile, active, contextpermeable architecture will be very useful. By allowing well-designed services and interfaces, it will be possible to continuously match users' expectations and activities resulting in greater integration and customization.
\end{abstract}

Keywords: ciber-physical systems, IS portfolio, process architecture, enterprise architecture, 4th industry.

\section{Introdução}

A tecnologia tem feito tanta diferença que a sua ligação com o capital humano tem feito emergir daí um enorme potencial. As tecnologias de informação (TI) constituem 
a plataforma para as empresas desenvolverem sistemas de informação (SI) capazes de lidar com novos requisitos de gestão. Como por exemplo a habilidade crescente para explorar enormes volumes de dados em grandes bases de dados (data warehouses), usando ferramentas avançadas para relacionar dados (data mining), e para responder a necessidades mais variadas. Os desafios atuais incluem novos modos de apresentar ou entregar produtos/serviços, como os que combinam características físicas com digitais. Estas tendências implicam mudanças nos modelos de negócio e interfaces de utilizador, levando a repensar a estratégia de negócio e inovação. Assim, as empresas deveriam organizar os seus SI/TI de modo a desenvolver novas soluções que melhorem a sua posição competitiva no mercado.

Daí que o presente trabalho pretenda discutir uma abordagem ao planeamento do portefólio de SI. Este conceito refere-se ao conjunto de ferramentas e metodologias de SI/TI de suporte ao modelo de negócio e sua dinâmica. Esta abordagem incentiva a atenção para a gestão adequada desse portefólio, que deve assentar numa seleção e alinhamento regulares com os objetivos de negócio.

\section{Inovação em Portugal}

\subsection{A economia Portuguesa}

Em Portugal as empresas (maioritariamente de pequena e média dimensão) investem pouco em I\&D (investigação e desenvolvimento) devido à sua menor capacidade financeira e organizacional. Assim, os SI são um importante recurso para a sua performance de negócio. Sobre os setores mais inovadores da economia Portuguesa que, de acordo com Sarkar (2014) tendem a estar mais orientados aos SI, o inquérito comunitário à inovação (CIS) fornece informação relevante. A União Europeia usa este instrumento para monitorizar o progresso da Europa em termos de inovação, o qual é conduzido por institutos de estatísticas nacionais. O CIS pretende recolher dados sobre inovação (de produto, processo, marketing e organizacional) diretamente das empresas. E explora como as empresas interagem com o seu ambiente externo de modo a obterem informação para novos projetos ou desenvolvimento de existentes.

Um CIS recente (CIS2012) revela que os setores mais inovadores em Portugal são de tipo: research-based (computação, engenharia civil, I\&D) ou knowledge-based (seguros, saúde) ou service-based (comércio). As fontes/agentes mais utilizadas pelo primeiro tipo são universidades, pelo segundo são fornecedores e pelo terceiro são as empresas do grupo. Os clientes são recursos importantes em todos os tipos de setores apontados, o que revela que as empresas Portuguesas geralmente usam informação e relações com os clientes para objetivos de inovação. Estes resultados estão de acordo com o enfoque crescente no conhecimento e nos serviços, para os quais o design de interfaces é um recurso interessante. Interfaces de utilizador flexíveis permitem expandir o portefólio de SI e melhorar a inovação e inteligência de negócio (BI) (Sánchez-González e Herrera, 2014).

O portefólio de SI em que as empresas Portuguesas devem incidir, uma vez que a maioria tem pequena ou média dimensão e uma cultura de serviço, inclui ferramentas tais como CRM (gestão da relação com o cliente), ERP (sistemas integrados de gestão), analítica de Big data e sistemas móveis (Fernandes, 2013). Estas ferramentas, combinadas em 
plataformas bem planeadas, contribuem para conceber e testar novos produtos/serviços, melhorar os processos existentes e criar novos processos. Dado o enorme potencial os gestores deveriam, não só estar familiarizados com essas tecnologias, mas também saber selecioná-las e alinhá-las com seus objetivos e envolvendo todos na organização.

\subsection{Tendências nos SI}

Muitas empresas Portuguesas têm usado sistemas financeiros que processam automaticamente faturas e relatórios a partir de folhas de dados (Fernandes, 2010). Contudo, face ao enorme volume de dados resultante de ter um website ou rede(s) social(is) associada(s), elas necessitam usar novas ferramentas para gestão big-data (Borrero e Gualda, 2013; Araújo e Cota, 2016). Um ERP pode ajudar, porque permite a integração de diferentes funções de negócio e dados, informando o tracking de produto, as preferências dos clientes, etc. Os fluxos de informação resultam mais rápidos e completos, contribuindo para um inventário consistente e uma resposta correta (Vasilev e Georgiev, 2003).

Os sistemas integrados permitem que a informação flua facilmente entre diferentes funções para desenvolver diferentes processos. A informação é então armazenada num único grande repositório (data warehouse), disponível a todas as unidades de negócio em todos os níveis de gestão. Todos os participantes podem ter a informação de que necessitam em tempo real (Laudon e Laudon, 2004). Por outro lado, as ferramentas de CRM consistem em funções analíticas para gerir a relação com os clientes e consolidar informação de diferentes fontes ou canais de comunicação. As empresas podem usar essa informação para adquirirem novos clientes, melhorarem seus produtos/serviços, personalizarem-nos de acordo com preferências dos clientes, etc. Estas interessantes ferramentas podem estar online através de infraestruturas nuvem (cloud) e advirem móveis e sensíveis ao contexto através de redes wireless e sensores.

Estes recursos e a sua interoperabilidade constituem uma área chave a ser explorada pelas empresas em diversos setores (saúde, turismo, banca, etc.). Porque a mesma permite inovar serviços, valorizar as preferências dos clientes e fornecer rápido conteúdo personalizado.

\subsection{Importância da IoT}

O potencial impressionante clould-wireless (da rede sem fios com a computação nuvem) requer uma abordagem flexível à arquitetura de processos nas empresas. O objetivo principal é gerir a aderência a novos modelos de negócio suportados por tecnologias como IoT (Internet-of-things), big-data e apps (aplicativos) móveis. Isto é importante pois os futuros fundos estruturais de investimento da UE (União Europeia) assentam em desenvolver e corresponder estas forças às necessidades de mercado (European Commission, 2016).

Uma rede de dispositivos inteligentes pode ser configurada para melhorar o funcionamento de qualquer negócio e seus serviços. Por exemplo, no turismo, pode proporcionar uma melhor assistência aos turistas através de apps móveis que guiam a experiência de visitar um lugar, pela coordenação inteligente dos objetos desse lugar. Isso contribui para melhorar a impressão do turista e a qualidade da sua experiência. 
Essas orientações móveis podem incluir detalhes sobre atrações locais, alojamento, restaurantes e assistência ao longo do resto da viagem.

Também o empowerment dos trabalhadores (ou dos clientes) é possível com as plataformas móveis, o que é relevante em atividades críticas como na área da saúde. Sensores interconectados, quer vestidos, quer embutidos no ambiente envolvente, tornam possível a recolha de informação relevante (Hassanalieragh et al., 2015; Niewolny, 2013; Tyagi et al., 2016).

\section{Gestão do portefólio de SI}

Existe um problema comum nas empresas, relacionado com o crescente volume de dados e de SI não integrados, afetando a performance da empresa. Uma razão é o facto de que o potencial de novas tecnologias (tais como clouds, websites, IoT, etc.) está longe de ser totalmente explorado. Isto levanta a necessidade da gestão de um portefólio adequado de SI na organização. Um objetivo disso é responder a necessidades de negócio de um modo inteligente e inovador (Robbins, 2006).

Tal como inicialmente referido, o presente trabalho incide em delinear uma abordagem ao portefólio de SI. Este conceito refere-se ao conjunto de ferramentas e metodologias (ERP, clouds, social media, wireless, apps, etc.) de suporte ao modelo de negócio. Esta abordagem destaca a necessidade de uma gestão adequada desse portefólio, orientada para a seleção e alinhamento regular com as perspetivas de negócio (figura 1).

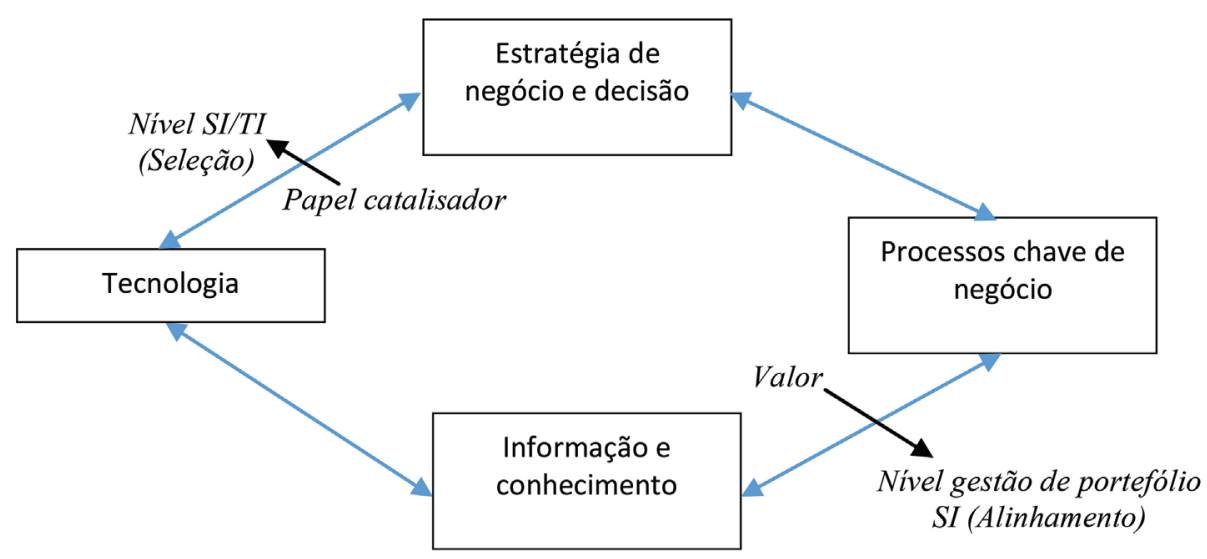

Figura 1 - Portefólio de SI e seu papel estratégico

Devidamente planeado e alinhado, ao nível dos processos, um portefólio de SI pode ser assim um catalisador (ou controlador) de valor para o modelo de negócio (Vanti et al., 2018).

\subsection{Catalisador de valor: Como?}

Em certas indústrias os SI têm sido diferenciadores estratégicos por vários anos (Laudon e Laudon, 2004). Assim, o desenvolvimento de produtos e serviços deve considerar as 
oportunidades e limitações tecnológicas (papel catalisador). Um portefólio de SI que sustenta conhecimento e inteligência para os processos chave é crucial para o sucesso do negócio (valor acrescentado).

Uma datawarehouse é a base de dados mais apropriada, porque normalmente contém dados de todas as funções da organização. A figura 2 ilustra o caso Kraft foods como exemplo disso (Kraft, 2002).

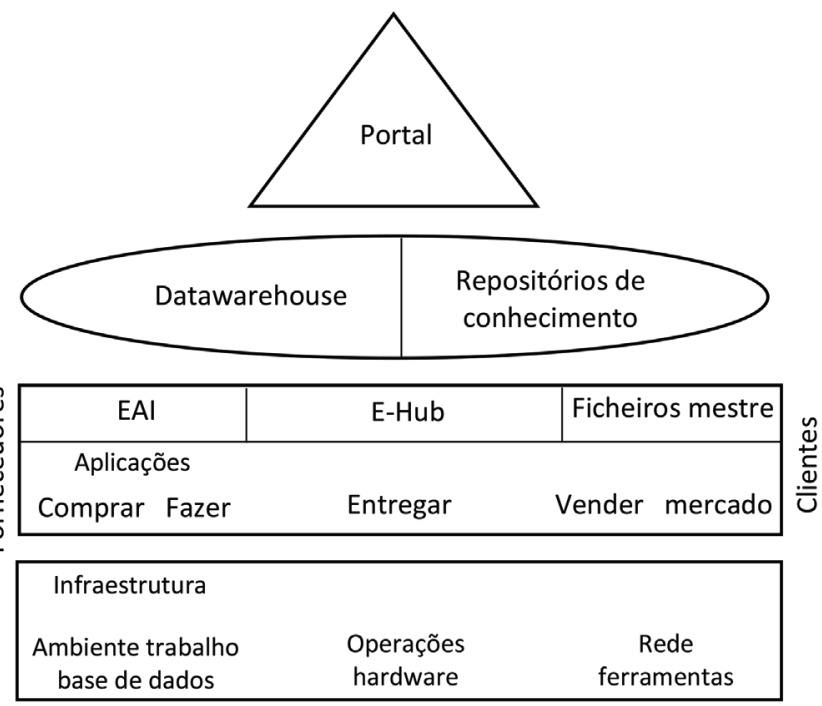

Figura 2 - Portefólio de SI no grupo Kraft foods

Muito se fala sobre ter uma datawarehouse (DW), datamining (DM) e data analytics (DA). Alguns autores, no entanto, sugerem que as expetativas associadas são mais altas do que aquilo que se retira efetivamente dessas ferramentas e técnicas. Embora a DA permita identificar vantagens competitivas, algumas destas não são exequíveis devido às grandes mudanças que envolvem (Laudon e Laudon, 2004; Ross et al., 2013). As empresas devem começar por conhecer bem os dados e ferramentas que já possuem, pois poucas tiram pleno partido das mesmas para responder a desafios do mercado. Isso requer uma prática diária, assente numa cultura baseada nos dados, envolvendo toda a organização.

As organizações precisam tirar vantagem dos seus dados operacionais e tomar decisões diárias com base neles. E daí, conceder acesso a todos os níveis da organização em tempo real, definir procedimentos e revê-los numa base regular. Desse modo estarão a decidir com base na evidência dos dados (Ross et al., 2013) e, se o fizerem, darão poder aos seus trabalhadores.

\section{Importância da arquitetura empresarial}

Os analistas de SI são aqueles que mais lidam com a necessidade de haver diálogo com as entidades que requerem um suporte adequado de SI. Nesse sentido, usam 
modelos para representar a realidade que precisam avaliar, tal como um desenho estruturado ou arquitetura para rapidamente explorar uma solução (Ambler et al., 2005). Dada a velocidade de mudança que os SI instilam nos processos de negócio, devem ser então avaliados à luz da arquitetura de processos (Spewak e Hill, 1992), e mais amplamente da arquitetura empresarial. Isso consiste em definir e compreender os diferentes elementos que constituem a organização, seu modelo de negócio e processos, bem como estes se relacionam dentro e fora dela (Sousa et al., 2006). A gestão de portefólio dos atuais SI/TI requer uma crescente necessidade de modelizar os fluxos de dados e processos, para melhor discernir sua seleção e alinhamento com as perspetivas de negócio.

\subsection{Arquitetura aberta}

Existem vários modelos de arquitetura empresarial, cada um envolvendo uma abordagem ligeiramente diferente. Mas em cada modelo prevalece uma transição explícita do negócio para os SI, ou seja, dos objetivos e requisitos para as aplicações e sistemas. Contudo, essa transição pode ser menos explícita devido a requisitos e métodos abstratos do modelo de negócio ditados pelas ferramentas de TI. A conectividade móvel e extensiva está revelando que a fronteira entre a arquitetura empresarial (AE) e as aplicações tende para uma área (em vez de uma linha). Assim, uma maior flexibilidade é essencial para obter resultados mais diferenciadores (Nolle, 2016).

De acordo com Palli e Behara (2014), sem uma AE aberta haverá problemas tais como: falta de flexibilidade das soluções implantadas; inconsistências; redundâncias; não adoção de tecnologias emergentes; falta de integração e inter-operacionalidade de aplicações; etc.

Porque a AE tradicional émais centrada na estrutura (framework-centered) do que orientada pelas ferramentas (tool-driven). A maior fatia da sua função é centrada na tecnologia. Enquanto a AE aberta (NGEA) é centrada no negócio, e daí é global, ágil e contínua.

A abordagem da AE tradicional tende a mudar para a NGEA (ou arquitetura digital), a qual envolve capacidades de rede tais como: interagir com utilizadores e outros agentes através de social media; globalização (empresa 'sem fronteiras'); inovação de produto/ serviço (inovação aberta e virtual); colaboração (empregados no processo de decisão, trabalho móvel); e flexibilidade (para escolher tecnologias, infraestrutura e aplicações). Dessa maneira, as organizações adotam as últimas capacidades digitais como social web, service-oriented architecture (SOA), big-data analytics, cloud computing, virtualização, IoT, etc. (Palli e Behara, 2014) - figura 3.

Por sua vez, os resultados da AE devem integrar-se com o planeamento de negócio e o enfoque no modelo de negócio, definindo métricas de retorno. E a definição do retorno da $\mathrm{AE}$ não deveria estender-se por anos, mas sim devolver valor ao negócio em meses ou semanas.

Atualmente os SI tendem a ser cada vez mais ciber-físicos para corresponderem ao desafio da 'transformação digital' das empresas e das economias. Tais sistemas híbridos permitem descentralizar e monitorizar processos físicos entre os colaboradores da cadeia de fornecimento, independentemente do seu lugar e função. Estes sistemas tornam-se 
IoT, comunicando e cooperando entre si e com os humanos em tempo real via wireless. Assim a perceção (ou sensibilidade) ao contexto é mais uma variável a contemplar.

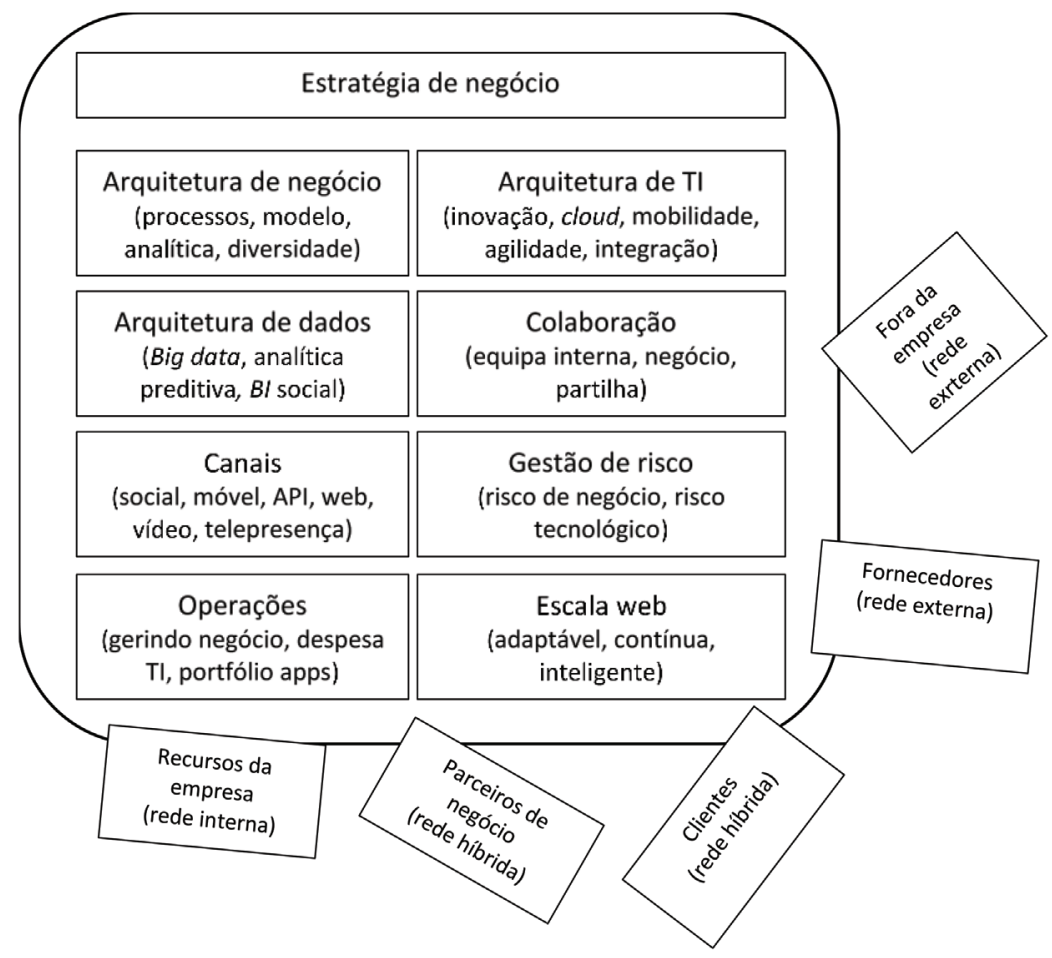

Figura 3 - Modelo de AE de próxima geração (NGEA)

\subsection{O contexto nos processos ciber-físicos}

A figura 4 ilustra a importância da NGEA para uma correspondência de perceção de contexto: a perceção e experiência do utilizador têm a ver com as expetativas do mesmo as quais a empresa e seus SI, se forem sensíveis ao contexto, capturam por input sensorial (sensores). Por isso, uma AE aberta deve considerar a abordagem do utilizador dirigir a reação dos sistemas empresariais (Schmidt, 2013).

Uma grande variedade de sensores pode ser usada para captar informação contextual. Alguns exemplos de sensores usados são: GPS (para localização, velocidade); luz e visão (para detetar objetos, atividades); microfones (para dados sobre ruído, falas); giroscópios (para movimento, orientação); sensores magnéticos (para determinar orientação); sensores táteis (para detetar interação com o utilizador); sensores de temperatura (para avaliar ambientes); etc. (Schmidt, 2013).

Outros sensores servem para detetar o contexto fisiológico do utilizador, como por exemplo para captar respostas da pele. As suas medições podem ser usadas para avaliar reações da pele (por exemplo surpresa, etc.). Estes tipos de sensores disponíveis podem ser usados para alimentar os SI empresariais com informações de contexto. 


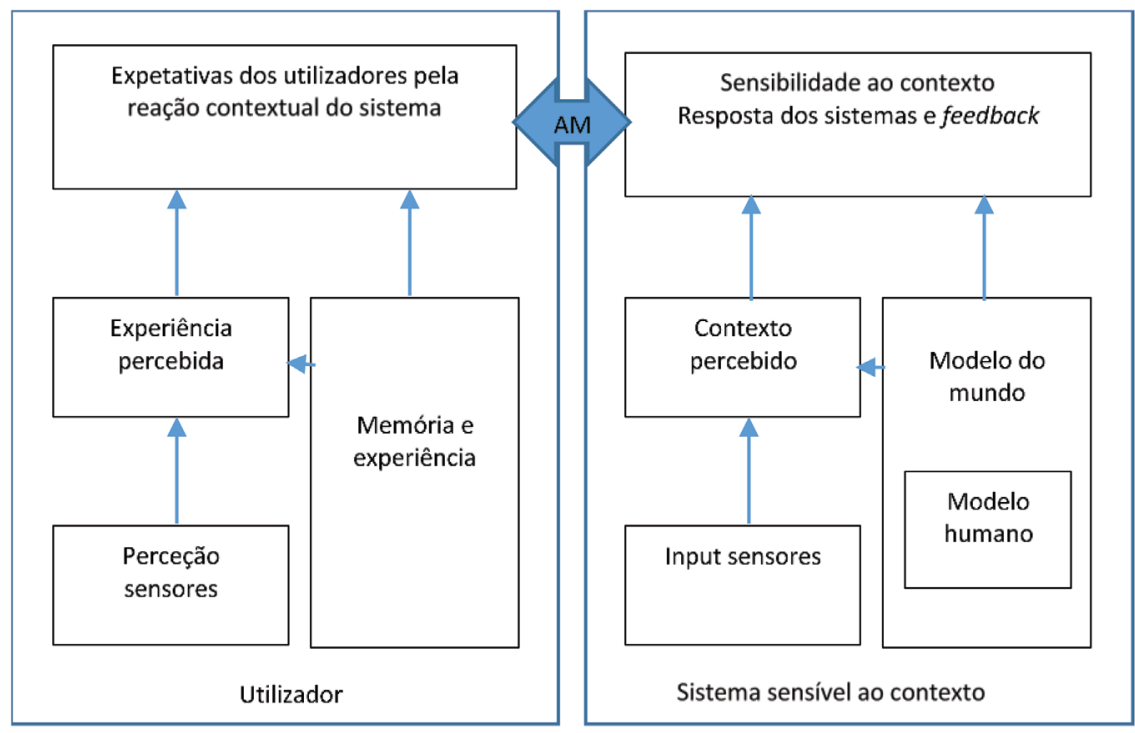

Figura 4 - Modelo de NGEA com correspondência de perceção

Esta variedade de tecnologias (cloud, wireless, sensores, etc.) permite assim criar sistemas que podem atuar consoante diferentes contextos. Se forem bem delineados, eles corresponderão melhor às expetativas dos utilizadores no seu contexto. É também essencial que os utilizadores compreendam o comportamento variável das aplicações e saibam como usá-las nas situações em que se encontram.

\subsection{Caso na indústria 4}

Uma das grandes razões do progresso dos sistemas ciber-físicos é a sua utilização em áreas em expansão como a realidade aumentada e a indústria 4. A procura por produtos/ serviços personalizados cresceu significativamente nos últimos anos, o que exigiu profundas alterações nos processos de produção da indústria tradicional. De forma a passarem a existir linhas de produção dinâmicas e flexíveis, tais sistemas permitem estabelecer linhas capazes de produzir produtos personalizados em larga escala. A adaptação dos formatos de produção foi um desafio para os produtores, cujos custos elevados e soluções limitadas tornaram-se um problema que vários estudos tentaram resolver.

O avanço tecnológico permitiu explorar soluções viáveis mais simples e eficazes. Isto consegue-se através da integração de tecnologias da informação com a engenharia de controlo nos processos de produção, assentando em IoT e sistemas ciber-físicos (figura 5). No caso estudado por Ramião (2017), propõe-se a utilização de uma arquitetura modular para gerir e controlar descentralizadamente os sistemas de produção com tecnologia plug\&produce. Por serem sistemas que integram o mundo físico com aplicações computacionais, permitindo criar módulos (grupos de aplicações) com capacidade de funcionamento autónomo e distribuído, há a necessidade para isso de middleware para sincronização (como por exemplo o CiberSens). 


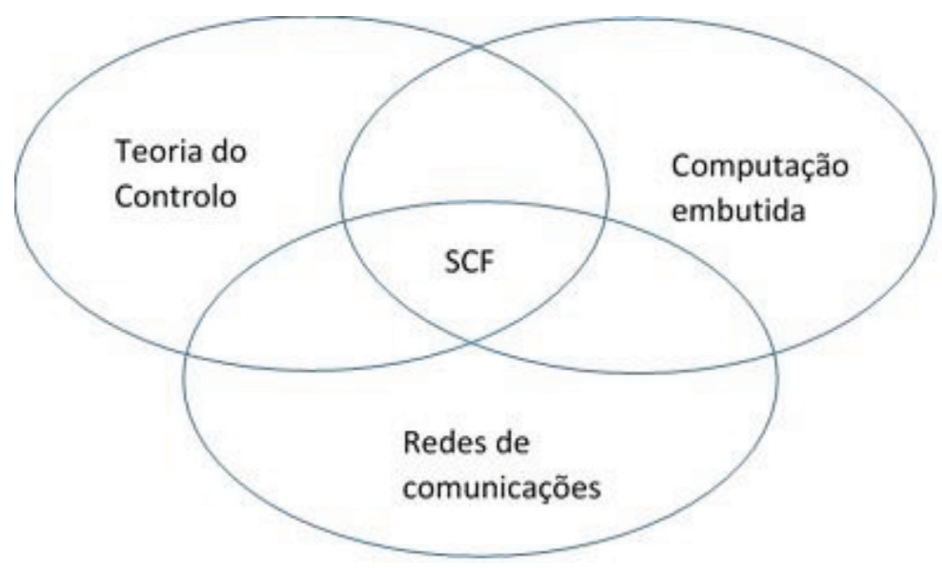

Figura 5 - Áreas integrantes dos sistemas ciber-físicos (SCF)

A utilização de dispositivos IoT e sistemas multiagentes permitem um controlo dinâmico e flexível de linhas de montagem mantendo um custo relativamente baixo. A arquitetura proposta por Ramião (2017) responde afirmativamente à questão de ser possível desenhar um módulo de produção a um custo reduzido, capaz de abstrair um componente de produção e oferecer uma solução de controlo flexível. As características dessa arquitetura - como modularidade, capacidade de plug\&produce, e sistema descentralizado e hierárquico - permitem que um sistema possa ser reorganizado de acordo com as necessidades de produção. Isso só é possível devido à utilização de uma arquitetura ciberfísica de produção modular onde cada bloco do sistema (módulo) pode ser realocado ou substituído de acordo com as necessidades correntes. Mais uma vez o recurso prévio à arquitetura aberta de processos será uma mais-valia neste tipo de projetos.

Os sistemas tradicionais de controlo industrial normalmente têm um preço mais elevado e as características dos sistemas centralizados, dificultando muito a produção de produtos altamente personalizáveis.

\section{Conclusão}

A natureza dos processos de negócio está a mudar, devido frequentemente à velocidade de mudança das tecnologias de informação. Isso tem trazido muitos desafios às organizações e sociedade. Tais desafios culminam em dois principais vetores (drivers): mobilidade e conectividade. Estes levam à necessidade de um modelo de trabalho (arquitetura) para planear e facilitar o alinhamento com as perspetivas de negócio e sistemas existentes.

Uma arquitetura de processos aberta da empresa e do seu plano, fácil de compreender e comunicar, pode ajudar a identificar a estrutura consistente com a missão da empresa, objetivos e produtos/serviços pretendidos. É especialmente desenvolvida com base em objetos como: processos (funcionais e transversais, internos e externos); recursos (funcionais e transversais, internos e externos); contexto e outputs (internos e externos). 
Contudo, devido à heterogeneidade daqueles objetos e dos dados que os caracterizam, dois dos aspetos mais prementes hoje são a sua integração e conectividade. Várias ferramentas da chamada transformação digital (IoT, sistemas ciber-físicos e móveis) podem contribuir para alcançar elevados níveis de flexibilidade e personalização com as capacidades da cadeia de valor. E podem assim ajudar na criação de novas fontes de inovação e retorno por suportarem a produção inteligente de produtos/serviços.

Dado o seu enorme potencial, que pode levar à reconfiguração dos modelos de trabalho e negócio, os gestores devem estar familiarizados com tais ferramentas. E também estar envolvidos com sua seleção, adoção e alinhamento desde o início.

Este artigo é financiado pelo fundo nacional da FCT - Fundação para a Ciência e a Tecnologia através do projeto UID/SOC/04020/2013.

\section{Referências}

Ambler, S., Nalbone, J., \& Vizdos, M. (2005). The Enterprise Unified Process: Extending the Rational Unified Process. New Jersey: Prentice Hall.

Araújo, V., \& Cota, M. (2016). Software como um serviço: Uma visão holística. RISTI Revista Ibérica de Sistemas e Tecnologias de Informação, (19), 145-157. Doi: $10.17013 /$ risti.19.145-157.

Borrero, J., \& Gualda, E. (2013). Crawling big data in a new frontier for socioeconomic research: Testing with social tagging. Journal of Spatial and Organizational Dynamics, 1(1), 3-24.

European Commission (2016). Smart regions conference. Disponível em http://ec.europa.eu/regional_policy/en/conferences/smart-regions/ (acedido em 23 de Outubro de 2017).

Fernandes, S. (2010). Critical information as a success factor in organizations: Objective and methodological approach. Spatial and Organizational Dynamics Discussion Papers, 2, 69-82.

Fernandes, S. (2013). Aligning technology with business: A continuous effort. Spatial and Organizational Dynamics Discussion Papers, 12, 26-37.

Hassanalieragh, M., Page, A., Soyata, T., Sharma, G., Aktas, M., Mateos, G., Kantarci, B., \& Andreescu, S. (2015). Health monitoring and management using internet-of-things (IoT) sensing with cloud-based processing: Opportunities and challenges. Paper presented at the IEEE International Conference on Services Computing, New York, USA.

Kraft (2002). Information systems portfolio management. Disponível em http://www.kraftfoodsgroup.com/home/index.aspx (acedido em 12 de Junho de 2017).

Laudon, C., \& Laudon, J. (2004). Management Information Systems: Managing the Digital Firm. Boston: Prentice Hall.

Niewolny, D. (2013). How the internet of things is revolutionizing healthcare. Disponível em https://cache.freescale.com/files/corporate/doc/white_paper/ IOTREVHEALCARWP.pdf (acedido em 21 de Outubro de 2017). 
Nolle, T. (2016). Enterprise architecture model helps to maximize mobile empowerment. Disponível em http://searchsoa.techtarget.com/tip/Enterprise-architecturemodel-helps-to-maximize-mobile-empowerment?utm_medium=EM\&asrc=EM_ NLN_6580939o\&utm_campaign $=20161007$ Enterprise\%20architecture\%20 holds\%2othe\%2osecret\%2oto\%20mobile_fchurchville\&utm source $=$ NLN\&track $=$ NL-1806\&ad $=910397 \& s r c=910397$ (acedido em 21 de Outubro de 2017).

Palli, P., \& Behara, G. (2014). Enterprise architecture: A practitioner view. Disponível em https://blog.opengroup.org/2014/o9/17/enterprise-architecture-a-practitionerview/ (acedido em 11 de Setembro de 2017).

Ramião, A. (2017). Sistema ciber-físico de produção modular usando Raspberry Pi. Dissertação de Mestrado, Faculdade de Ciências e Tecnologia, Universidade Nova de Lisboa.

Robbins, S. (2006). Organizational Behavior. Canada: Prentice Hall.

Ross, J., Beath, C., \& Quaadgras, A. (2013). You may not need big data after all. Disponível em https://hbr.org/2013/12/you-may-not-need-big-data-after-all (acedido em 11 de Setembro de 2017).

Sánchez-González, G., \& Herrera, L. (2014). Effects of customer cooperation on knowledge generation activities and innovation results of firms. Business Research Quarterly, 17(4), 292-302. Doi: 10.1016/j.brq.2013.11.002.

Sarkar, S. (2014). Entrepreneurship and Innovation. Portugal: Escolar Editora.

Schmidt (2013). Context-aware computing. Disponível em https://www.interactiondesign.org/literature/book/the-encyclopedia-of-human-computer-interaction2nd-ed/context-aware-computing-context-awareness-context-aware-userinterfaces-and-implicit-interaction (acedido em em 23 de Outubro de 2017).

Sousa, P., Caetano, A., Vasconcelos, A., Pereira, C., \& Tribolet, J. (2006). Enterprise architecture modeling with the unified modeling language, In P. Ritten (Ed.), Enterprise modelling and computing with $U M L$. Hershey: IRM Press.

Spewak, S., \& Hill, S. (1992). Enterprise Architecture Planning: Developing a Blueprint for Data, Applications and Technology. New Jersey: John Wiley \& Sons.

Tyagi, S., Agarwal, A., \& Maheshwari, P. (2016). A conceptual framework for IoT-based healthcare system using cloud computing. Paper presented at the International Conference on Cloud System and Big Data Engineering, Noida, India.

Vanti, A., Solana-González, P., \& Seibert, R. (2018). Gobernanza corporativa y gobernanza corporativa de TI utilizando Analytic Hierarchy Process en la creación de valor. RISTI - Revista Ibérica de Sistemas e Tecnologias de Informação, (27), 86-108. Doi: 10.17013/risti.27.86-108.

Vasilev, J., \& Georgiev, G. (2003). Tendencies in the development of ERP systems. Paper presented at the International Conference on Computer Systems and Technologies, Sofia, Bulgaria. 